\title{
Tunable Cavity Optomechanics with Ultracold Atoms
}

\author{
T.P. Purdy ${ }^{1}$, D.W.C. Brooks ${ }^{1}$, T. Botter ${ }^{1}$, N. Brahms ${ }^{1}$, Z.-Y. Ma ${ }^{1}$ and D.M. Stamper-Kurn ${ }^{1,2}$ \\ ${ }^{1}$ Department of Physics, University of California, Berkeley CA 94720, USA \\ ${ }^{2}$ Materials Sciences Division, Lawrence Berkeley National Laboratory, Berkeley, CA 94720, USA
}

(Dated: November 3, 2018)

\begin{abstract}
We present an atom-chip-based realization of quantum cavity optomechanics with cold atoms localized within a Fabry-Perot cavity. Effective sub-wavelength positioning of the atomic ensemble allows for tuning the linear and quadratic optomechanical coupling parameters, varying the sensitivity to the displacement and strain of a compressible gaseous cantilever. We observe effects of such tuning on cavity optical nonlinearity and optomechanical frequency shifts, providing their first characterization in the quadratic-coupling regime.
\end{abstract}

Experimental realizations of cavity optomechanics, wherein the motion of a mechanically compliant cantilever is measured by its interaction with an electromagnetic resonator, serve as paradigms for understanding open quantum systems and the limits of quantum measurement [1]. In most realizations, cavity optomechanical phenomena, such as optical cooling [2 5 ] and confinement [6, 7. of the cantilever or optical nonlinearity [8, 9] and squeezing 10, 11, arise from the dominant linear coupling between cavity photons and the cantilever position. Recent experiments on thin SiN membranes positioned within a Fabry-Perot cavity [12, 13] have highlighted the new capabilities afforded by quadratic optomechanical coupling, such as measurement of the cantilever energy and of phonon shot noise [14].

Here, we demonstrate a tunable cavity optomechanical system constructed by integrating a microfabricated atom chip with a Fabry-Perot optical resonator. We tune the optomechanical sensitivity to the displacement and the strain of an atomic ensemble, arising from linear and quadratic optomechanical coupling, respectively, by positioning cold atoms with nm-scale precision within the resonator mode. The ensemble thereby serves as the quantum analogue of the SiN membranes used in recent experiments [12, 13. We study effects of tunable coupling on optomechanical bistability and the optomechanical frequency shift, providing the first characterization of optomechanical effects in the quadratic-coupling regime. The agreement between our measurements and theory establishes the equivalence of cavity optomechanical systems using either solid- or gas-phase cantilevers.

We begin by adapting recent theoretical descriptions of cavity optomechanics using cold atoms 15 to highlight the new capabilities presented in this work. Consider the motion of $N$ identical atoms along the axis $(\hat{z})$ of a FabryPerot optical resonator and confined within a harmonic potential with mechanical frequency $\omega_{z}$ and centered at position $z_{0}$. The interaction of atom $i$ at $z_{i}=z_{0}+\delta z_{i}$ with the cavity field is characterized by the angular frequency $g\left(z_{i}\right)=g_{0} \sin \left(\phi_{0}+k_{p} \delta z_{i}\right)$, where $\phi_{0}=k_{p} z_{0}, k_{p}$ is the resonant cavity wavevector and $g_{0}$ is determined by the cavity mode volume, the optical frequency, and atomic dipole matrix elements. Assuming the detuning
$\Delta_{\text {ca }}=\omega_{c}-\omega_{a}$ between the cavity $\left(\omega_{c}\right)$ and the atomicelectronic $\left(\omega_{a}\right)$ resonance frequencies is large, we retain the dispersive atoms-cavity interaction, and expand to second order in the Lamb-Dicke parameters $k_{p} \delta z_{i} \ll 1$, obtaining the following interaction Hamiltonian:

$$
\mathcal{H} \simeq\left[\hbar \Delta_{N}^{(0)}-F Z_{\mathrm{cm}} \sin 2 \phi_{0}-F k_{p}\left(Z_{\mathrm{cm}}^{2}+\sigma^{2}\right) \cos 2 \phi_{0}\right] \hat{n} .
$$

Here, the zero-order term defines the static dispersive shift of the cavity resonance, $\Delta_{N}^{(0)}=N\left(g_{0}^{2} / \Delta_{\text {ca }}\right) \sin ^{2} \phi_{0}$, with all atoms held at the trap center. The next term describes linear optomechanical coupling to the ensemble center of mass $Z_{\mathrm{cm}}$, acted upon by the per-photon force defined as $F=N \hbar k_{p} g_{0}^{2} / \Delta_{\text {ca }}$. The second-order term describes quadratic optomechanical coupling. We identify both a quadratic sensitivity to the cantilever mode and also a coupling to the position variance $\sigma^{2}$ of the atomic medium. The relative strength of the linear and quadratic couplings is controlled by the position of the ensemble along the cavity axis.

In previous experiments [9, 16], the broad spatial extent of the atomic ensemble suppressed the quadratic optomechanical coupling, while maintaining a linear coupling to phonon-like perturbations of the trapped gas. Here, we overcome this limitation by localizing the gas within a sub-micron region positioned variably along the cavity axis. Such localization is achieved using a combination of magnetic and optical traps produced using an atom chip (Fig. 1). This chip is fabricated by embedding $75-\mu \mathrm{m}$-thick electroplated copper electromagnet wires into a $500 \mu \mathrm{m}$ thick silicon substrate. These electromagnets are used to trap ${ }^{87} \mathrm{Rb}$ atoms and to transport them $2.2 \mathrm{~cm}$ along the chip surface into the modevolume of a Fabry-Perot optical resonator, formed by curved mirrors placed above and below the chip surface. At the location of the cavity, the substrate is thinned to $100 \mu \mathrm{m}$, allowing the mirrors to be spaced by $250 \mu \mathrm{m}$, and a $200 \times 400 \mu \mathrm{m}$ rectangular hole is etched through the chip to allow the cavity field to propagate unobstructed.

The cavity, with Gaussian mode waist of $25 \mu \mathrm{m}$, is stabilized with one of its $\mathrm{TEM}_{00}$ resonances near the ${ }^{87} \mathrm{Rb} \mathrm{D} 2$ transition, at wavenumber $k_{p}=2 \pi /(780 \mathrm{~nm})$. For this mode, the cavity finesse of $1.7 \times 10^{5}$ results 


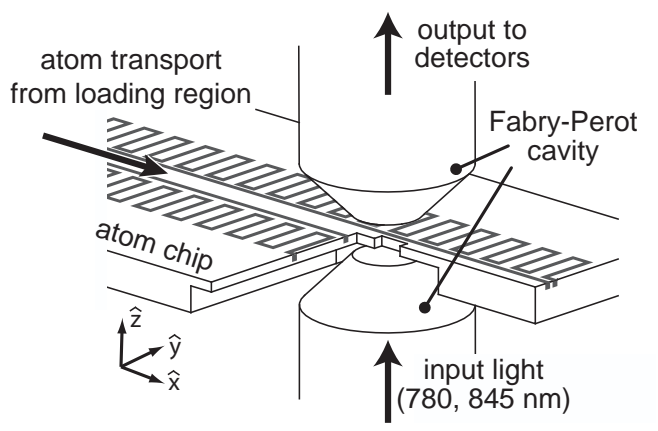

FIG. 1. An atom chip is used to transport and position atoms within a Fabry-Perot cavity, where they act as a compressible cantilever for studies of cavity optomechanics. A cutaway cross section shows the profile of the chip, with copper wires shown in gray.

from transmissions of 1.5 and $12 \mathrm{ppm}$ through the input and output mirrors, respectively, and additional losses of $26 \mathrm{ppm}$. An atom at the antinode of the cavity field, driven at the atomic resonance, experiences a coupling of $g_{0}=2 \pi \times 13.1 \mathrm{MHz}$, giving a single-atom cooperativity of $g_{0}^{2} / 2 \kappa \Gamma=16$ where $\kappa=2 \pi \times 1.8 \mathrm{MHz}$ and $\Gamma=2 \pi \times 3$ $\mathrm{MHz}$ are the cavity and the atomic half line-widths, respectively.

Once within the cavity, the magnetically trapped gas, with rms width less than $400 \mathrm{~nm}$ along the cavity axis, is transferred into the wells of a one-dimensional optical lattice trap formed by light with wavenumber $k_{t}=2 \pi /(845 \mathrm{~nm})$ driving a second $\mathrm{TEM}_{00}$ cavity mode [15. 17. This transfer is accomplished by ramping the optical lattice trap depth to $10 \mu \mathrm{K}$ before decompressing and switching off the magnetic trap. The lattice depth is lowered to $1.5 \mu \mathrm{K}$ to cool the atomic gas further, and then raised to provide a tunable axial oscillation frequency. The wells of this lattice trap serve as the near-harmonic mechanical potential for the several-thousand-atom ensembles used in our realization of cavity optomechanics. Time-of-flight temperature measurements on atoms released from the lattice imply an axial ground state occupation of $>90 \%$. Thus, our system realizes tunable cavity optomechanics in the quantum regime.

The position of the ensemble within the resonator is visible in the atom-induced shift of the cavity resonance, measured via the transmission of a low-power probe beam swept across the cavity resonance (Fig. 2). As expected, this frequency shift oscillates sinusoidally with the ensemble position with a spatial period $\pi /\left(k_{p}-k_{t}\right)=5 \mu \mathrm{m}$.

The contrast of this oscillation provides information on the distribution of atoms within the intracavity optical lattice. Assuming the axial density distribution of atoms within a lattice site to be Gaussian with rms width $\sigma=$ $\sqrt{\hbar / 2 m \omega_{z}}$, atoms in a single lattice site shift the cavity

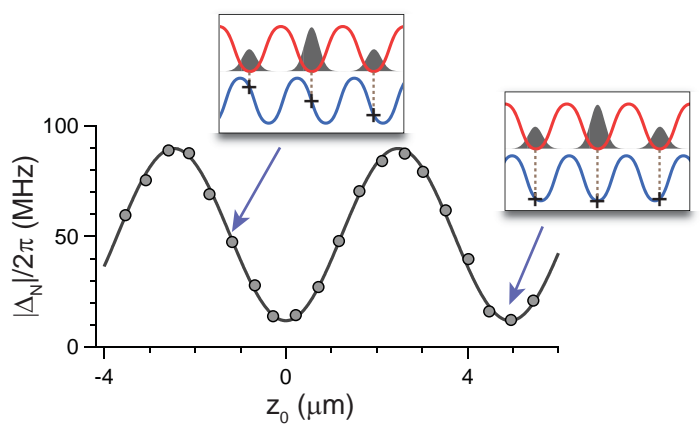

FIG. 2. (Color) The atom-induced shift of the cavity resonance at low probe power varies sinusoidally (fit shows contrast of 0.77 ) with the central atomic position $z_{0}$. Panels show estimated atomic distributions (gray), relative to the lattice trap potential (red, top) and cavity probe intensity (blue, bottom), at maximum linear (left) or quadratic (right) coupling. Here $N=3500, \Delta_{\text {ca }} / 2 \pi=-6 \mathrm{GHz}, \omega_{z} / 2 \pi=70 \mathrm{kHz}$.

resonance by [18]

$$
\Delta_{N}=N \frac{g_{0}^{2}}{2 \Delta_{\text {ca }}}\left(1-e^{-2 k_{p}^{2} \sigma^{2}} \cos 2 \phi_{0}\right) .
$$

The measured contrast is lower than this single-site limit, indicating that atoms are distributed among neighboring wells of the optical lattice. Assuming a Gaussian distribution of atoms among wells reduces the contrast by $e^{-2\left(k_{p}-k_{t}\right)^{2} \Sigma^{2}}$, we estimate the atoms to be divided among wells spanning $\Sigma \lesssim 400 \mathrm{~nm}$ along the cavity axis; that is, the ensemble is not confined to a single lattice site, though the majority of atoms occupy no more than two sites. We nevertheless achieve fine control of the optomechanical coupling owing to the near-equivalence of neighboring lattice sites, between which $\phi_{0}$ varies only by $0.26 \mathrm{rad}$. Thus, by using two similar wavelengths for the optical trapping and the probe light we parlay the moderate confinement of our chip-based magnetic trap to achieve sub-wavelength control of the gas-phase cantilever.

We use this tunable system to study the influence of variable linear and quadratic coupling parameters on two optomechanical effects. We consider first the cavityoptical nonlinearity that arises from steady-state modification of the atomic cantilever in response to the optical potential from an average of $\bar{n}$ probe photons within the cavity. This potential both displaces the center of mass and also changes the rms width of the compressible ensemble. These variations, ascribed to the linear and the quadratic optomechanical coupling, respectively, affect the atom-induced shift $\Delta_{N}(\bar{n})$ of the optical cavity resonance, now a function of $\bar{n}$, according to Eq. 1. The value of $\bar{n}$ is determined self-consistently from the Lorentzian cavity response function, $\bar{n}=\bar{n}_{\max }\left(1+\Delta^{2} / \kappa^{2}\right)^{-1}$ where $\Delta=\Delta_{\mathrm{pc}}-\Delta_{N}(\bar{n})$ is the detuning of the probe from the atoms-shifted cavity resonance, and $\bar{n}_{\max }$ characterizes 


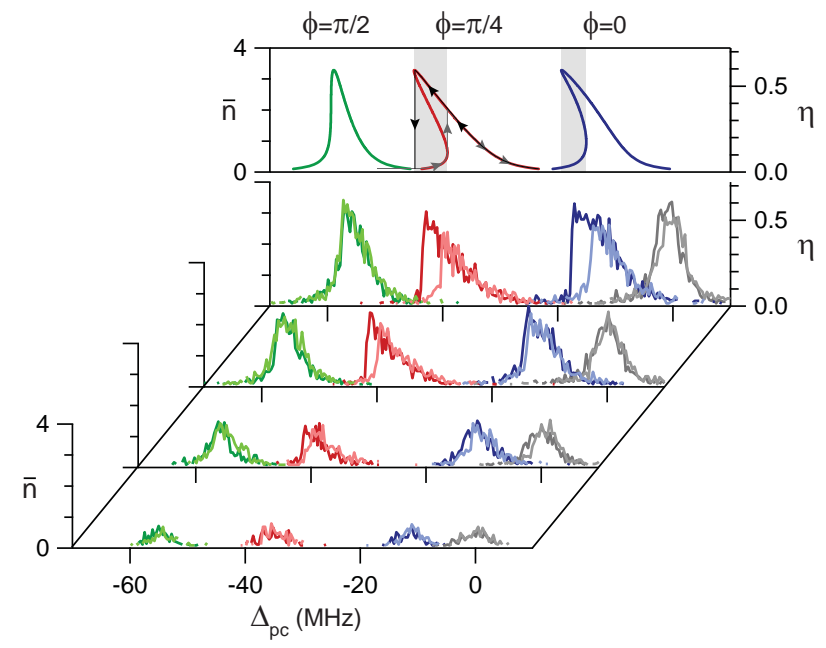

FIG. 3. (Color) Intracavity photon number $\bar{n}$ (or ratio of probe/trap curvatures $\eta$ ) for probe light swept with positive (lighter lines) or negative (darker lines) chirps. Measurements for four probe powers are shown, with $\phi_{0}=0$ (blue), $\pi / 4$ (red) or $\pi / 2$ (green). The horizontal scales are adjusted to account for a $2 \%$ loss of atoms between frequency sweeps. Gray traces show measurements for an empty cavity. Pronounced cavity nonlinearity is observed at $\bar{n} \simeq 1$ (input power around $5 \mathrm{pW}$ ). Here $N=5400, \Delta_{\text {ca }} / 2 \pi=-14 \mathrm{GHz}$, and $\omega_{z} / 2 \pi=32 \mathrm{kHz}$. Top graph shows self-consistent solutions for $\hbar$ calculated from known experimental parameters. Gray bars show regions of bistability in which two stable cavity and cantilever states are accessible at the same probe power and frequency. The expected line shape at $\phi=\pi / 2$ for probes with positive and negative chirps are indicated.

the incident probe power.

The cavity nonlinearity is characterized using two approaches. In the first, after preparing the atomic cantilever, we measure the cavity transmission of a constantpower probe as its frequency was swept slowly back and forth across the cavity resonance. The cavity transmission lineshapes show characteristic signs of cavity nonlinearity: displacement from the low-power cavity resonance, asymmetry, and, for sufficiently intense probe light, hysteresis signifying the mechanical bistability of the compressible atomic cantilever (Fig. 3).

As expected in the Lamb-Dicke regime $\left(k_{p} \sigma \ll 1\right)$, as the probe power is raised, the onset of cavity nonlinearity occurs first for atoms at the location of maximum linear optomechanical coupling $\left(\phi_{0}=\pi / 4\right)$. For atoms centered at the node and antinode of the probe field, residual linear coupling [19] provides a weaker nonlinear optical response at low probe powers. At higher probe powers, quadratic optomechanical coupling dominates and leads to a novel form of optomechanical cavity nonlinearity stemming from variation in the strain of the compressible cantilever. The strength of this strain-based nonlinearity depends on the ratio $\eta$ of the maximum curvatures of the probe-induced and mechanical potentials

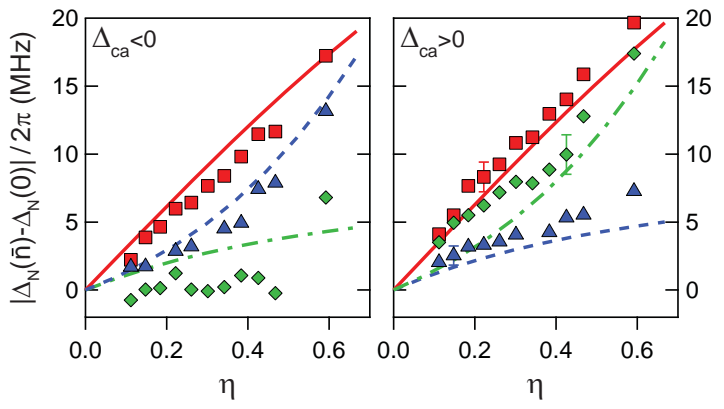

FIG. 4. (Color) Probe-induced shift of the cavity resonance vs. the probe/trap curvature ratio $\eta$, measured for $\phi_{0}=0$ (blue triangles, dashed line), $\phi_{0}=\pi / 4$ (red squares, solid line) and $\phi_{0}=\pi / 2$ (green diamonds, dot-dashed line). Representative statistical error bars are shown. The behaviours at the node and antinode are interchanged as the probe potential changes from attractive $\left(\Delta_{\mathrm{ca}} / 2 \pi=-8 \mathrm{GHz}\right)$ to repulsive $\left(\Delta_{\mathrm{ca}} / 2 \pi=+8 \mathrm{GHz}\right)$. The systematic discrepancy between measurements and theory (lines) arises from atom loss during the measurement; such heating and error are minimized at $\phi_{0}=0$.

acting on the atoms, given as $\eta=4 \bar{n} g_{0}^{2} \omega_{r} /\left(\Delta_{\text {ca }} \omega_{z}^{2}\right)$ where $\omega_{r}=\hbar k_{p}^{2} / 2 m$ is the recoil frequency. For $\Delta_{\text {ca }}<0$, the optical forces due to the probe field at $\phi_{0}=0$ subtract from the mechanical confinement of the trapping light, causing large shifts of the cavity resonance as the ensemble widens away from the field node; at $\eta=1$, the confinement to the field node is eliminated altogether. In contrast, for atoms positioned at the probe-field antinode $\left(\phi_{0}=\pi / 2\right)$, additional compression imparts only slight additional shifts to the resonance, reducing the nonlinear response.

A more quantitative measure of the cavity nonlinearity was obtained by a second technique, in which $\bar{n}$ was stabilized by feedback to the frequency of the cavity probe, fixing this frequency at a known amount from $\Delta_{N}(\bar{n})$. The cavity resonance shifts measured in this manner (Fig. 4) are in good agreement with calculations that account for the measured distribution of atoms among lattice sites.

We now turn to measurements of a dynamical optomechanical effect, the optomechanical frequency shift. We distinguish between two types of frequency shift. Considering again atoms trapped within a single lattice site, a "dynamic" frequency shift arises dominantly from linear optomechanical coupling. In a cavity probed away from the cavity resonance, the mean intracavity photon number varies linearly for small displacements of $Z_{\mathrm{cm}}$, yielding an additional "optical spring" 6, 20, with spring constant $K_{d}=\left[2 F^{2} \bar{n} \Delta /\left(\Delta^{2}+\kappa^{2}\right)\right] \sin \left(2 \phi_{0}\right)$. An additional "static" frequency shift arises from the quadratic optomechanical coupling, representing the added trap curvature from a constant-intensity intracavity probe field and quantified by the spring constant $K_{s}=2 k_{p} F \bar{n} \cos \left(2 \phi_{0}\right)$.

We note that the static frequency shift applies to the 


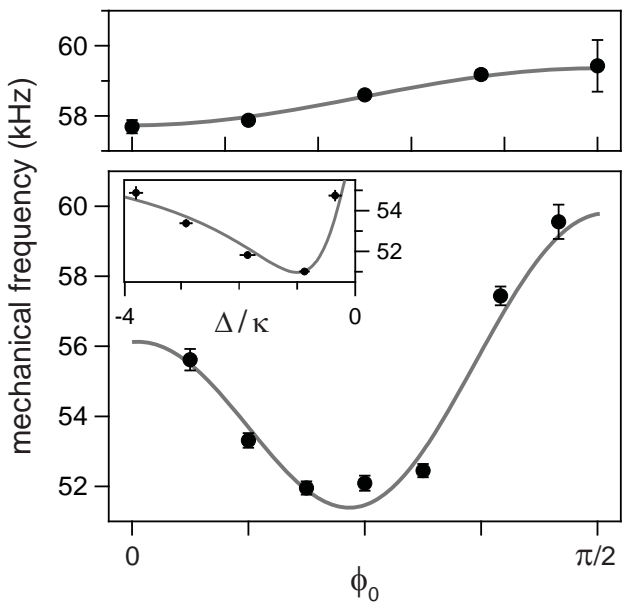

FIG. 5. Optomechanical frequency shifts. Top: the mechanical oscillation frequency determined via parametric heating reveals the static frequency shift from quadratic optomechanical coupling; here, $\Delta_{\text {ca }} / 2 \pi=20.1 \mathrm{GHz}, \omega_{z} / 2 \pi=58.5 \mathrm{kHz}$ and $\bar{n}=0.8$. Bottom: the oscillation frequency for the linearly coupled mode is derived from the cavity transmission intensity spectrum; here, $\Delta_{\text {ca }} / 2 \pi=40 \mathrm{GHz}, \omega_{z} / 2 \pi=58.5 \mathrm{kHz}, \bar{n}=3.5$ and $N=3750$. Inset shows this frequency at $\phi_{0}=\pi / 4$ for varying detuning $\Delta$ from the cavity resonance. Solid lines show predictions calculated with no free parameters.

motion of each individual atom in the ensemble, and therefore to all axial mechanical modes, including the linearly coupled mode. In contrast, the latter mode is uniquely influenced by the dynamic optomechanical frequency shift. This contrast allows us to distinguish experimentally between the two types of shift.

To observe the static frequency shift, we measured the resonant atomic oscillation frequency via parametric heating of the ensemble (Fig. 5). With probe light stabilized at fixed $\bar{n}$ and $\Delta$, we modulated the trap laser intensity at fixed frequency and then measured the fraction of atoms heated out of the optical trap by absorption imaging. Such modulation drives all mechanical modes, the influence of the linearly coupled mode being negligible with large $N$. Therefore, the dominant atom loss is observed at the frequency $2 \sqrt{\omega_{z}^{2}+K_{s} /(\mathrm{Nm})}$.

Alternately, we selectively detected the linearly coupled mode by monitoring temporal variations of the light transmitted through the cavity. Here, this mechanical mode is driven by technical and quantum noise of the probe light intensity [21. Our optomechanical system amplifies this noise with a frequency dependent gain [10, 11, 22, 23, that is visible in the power spectrum of the transmitted probe light. From a fit to this gain spectrum, we extracted the resonance frequency $\sqrt{\omega_{z}^{2}+\left(K_{s}+K_{d}\right) /(N m)}$ of the linearly coupled mode. The largest optomechanical frequency shift is observed at positions with maximal linear coupling $\left(\phi_{0}=\pi / 4\right)$ and at $\Delta=-\kappa$. The quantitative agreement with theoretical predictions with no free parameters is remarkable.

Our tunable cavity optomechanical system lies deeply in the quantum regime, as the cantilever is prepared near its ground state and its motion is dominated by quantum radiation pressure fluctuations. This capability should enable future studies of quantum optomechanical effects and of the role of linear and quadratic coupling in such phenomena. This platform also offers the possibility to enter the single-photon strong optomechanical coupling regime [15, 24, in which the nature of optomechanical effects remains poorly understood.

This work was supported by the NSF and the AFOSR. T.B. acknowledges support from Le Fonds Québécois de la Recherche sur la Nature et les Technologies, and D.M.S.-K. from the Miller Institute for Basic Research in Science.

* Present address: Shanghai Institute of Optics and Fine Mechanics, Chinese Academy of Sciences.

† dmsk@berkeley.edu

[1] T. Kippenberg and K. Vahala, Science 321, 1172 (2008).

[2] V. B. Braginsky and A. B. Manukin, Sov. Phys. JETP 25, 653 (1967).

[3] T. J. Kippenberg et al., Phys. Rev. Lett. 95, 033901 (2005).

[4] O. Arcizet et al., Nature 444, 71 (2006).

[5] S. Gigan et al., Nature 444, 67 (2006).

[6] B. S. Sheard et al., Phys. Rev. A 69, 051801(R) (2004).

[7] T. Corbitt et al., Phys. Rev. Lett. 98, 150802 (2007).

[8] A. Dorsel et al., Phys. Rev. Lett. 51, 1550 (1983).

[9] S. Gupta, K.L. Moore, K.W. Murch, and D.M. StamperKurn, Phys. Rev. Lett. 99, 213601 (2007).

[10] S. Mancini and P. Tombesi, Phys. Rev. A 49, 4055 (1994).

[11] C. Fabre et al., Phys. Rev. A 49, 1337 (1994).

[12] J. D. Thompson et al., Nature 452, 72 (2008).

[13] J. C. Sankey et al., preprint, arXiv:1002.4158

[14] A. A. Clerk, F. Marquardt, and J. G. E. Harris, preprint, arXiv:1002:3140.

[15] K. W. Murch, K. L. Moore, S. Gupta, and D. M. Stamper-Kurn, Nature Physics 4, 561 (2008).

[16] F. Brennecke, S. Ritter, T. Donner, and T. Esslinger, Science 322, 235 (2008).

[17] Y. Colombe et al., Nature 450, 272 (2007).

[18] The transverse extent of the ensemble only varies the effective cavity-atom coupling $g_{0}$, and not the contrast.

[19] For atoms in three neighboring wells with population ratios 1:2:1, linear coupling to the motions of atoms in the outer wells has relative strength $\sin ^{2}\left(2 \pi k_{p} / k_{t}\right) / 2=1 / 8$ compared to the maximum linear coupling; see Ref. [15].

[20] T. Corbitt et al., Phys. Rev. A 74, 021802(R) (2006).

[21] The small servo bandwidth of several $\mathrm{kHz}$ ensured that mechanical oscillations of the ensemble were not influenced by this feedback.

[22] F. Marino et al., Phys. Rev. Lett. 104, 073601 (2010).

[23] P. Verlot et al., Phys. Rev. Lett. 104, 133602 (2010).

[24] M. Ludwig, B. Kubala, and F. Marquardt, New Journal of Physics 10, 095013 (2008). 